\title{
PRÁTICA DE ENFERMEIRAS NA PROMOÇÃO DO ALEITAMENTO MATERNO DE ADOLESCENTES BRASILEIRAS
}

\author{
NURSING PRACTICES FOR BREASTFEEDING PROMOTION \\ IN BRAZILIAN ADOLESCENTS
}

\section{PRÁCTICA DE ENFERMERAS EN LA PROMOCIÓN DE LA LACTANCIA MATERNA DE ADOLESCENTES BRASILEÑAS}

\author{
Caroline CÂndido Garcia Leal ${ }^{\star}$ \\ Mariana de Oliveira Fonseca-Machado ${ }^{* *}$ \\ Lisiane Camargo Quialheiro de Oliveira *** \\ Juliana Cristina dos Santos Monteiro ${ }^{* * * *}$ \\ Adriana MORAES LeITE ${ }^{* * * *}$ \\ Flávia A. Gomes-Sponholz ${ }^{* * * * *}$
}

\begin{abstract}
RESUMO
Objetivo: Identificar a prática das enfermeiras atuantes na rede municipal de saúde de Ribeirão Preto, SP, relativa à promoção do aleitamento materno para gestantes e/ou mães adolescentes. Material e método: Pesquisa descritiva, qualitativa, realizada na rede básica de saúde de Ribeirão Preto, com 12 enfermeiras, em julho e agosto de 2009, por meio de entrevista semiestruturada e observação. Os dados foram analisados na modalidade temática da análise de conteúdo. Resultados: Identificamos as categorias "trabalho centrado na técnica, no recomendado e no biológico", "cotidiano do serviço de saúde na atenção às gestantes e/ou mães adolescentes" e "relação profissional de saúde e gestante e/ou mãe adolescente". As enfermeiras enfrentam o desafio da transformação da atenção centrada no procedimento em uma atenção focalizado no usuário. Ressaltam a preocupação em desenvolver uma relação de confiança, baseada na escuta e em incorporar questões relacionadas a dimensões sociais e subjetivas das gestantes e/ou mães adolescentes. Conclusão: São necessárias capacitação e educação permanentes em aleitamento materno, visando a um novo perfil de enfermeiros para a atenção integral das necessidades das adolescentes.
\end{abstract}

Palavras chave: Adolescente, assistência integral à saúde, aleitamento materno, enfermagem.

\footnotetext{
* Doutoranda da Escola de Enfermagem de Ribeirão Preto da Universidade de São Paulo. Professora adjunta da Universidade Paulista de Ribeirão Preto, SP, Brasil. CEP: 14098-780. E-mail: carolcgleal@gmail.com

${ }^{* *}$ Professora Adjunta, Departamento de Enfermagem, Universidade Federal de São Carlos - UFSCar. SP, Brasil. E-mail: mafonseca.machado@gmail.com. In memorian.

${ }^{* * *}$ Doutoranda da Escola de Enfermagem de Ribeirão Preto da Universidade de São Paulo. Ribeirão Preto, SP, Brasil. E-mail: lisiane.alves@usp.br

${ }_{* * * *}$ Doutora. Professora do Departamento de Enfermagem Materno-Infantil e Saúde Pública da Escola de Enfermagem de Ribeirão Preto da Universidade de São Paulo. Ribeirão Preto, SP, Brasil. E-mail: jumonte@eerp.usp.br

${ }_{* * * * *}$ Doutora. Professora do Departamento de Enfermagem Materno-Infantil e Saúde Pública da Escola de Enfermagem de Ribeirão Preto da Universidade de São Paulo. Ribeirão Preto, SP, Brasil. E-mail: drileite@eerp.usp.br.

${ }_{* * * * * *}$ Professora Associada do Departamento de Enfermagem Materno-Infantil e Saúde Pública da Escola de Enfermagem de Ribeirão Preto da Universidade de São Paulo. Ribeirão Preto, SP, Brasil. E-mail: flagomes@eerp.usp.br.
} 


\begin{abstract}
Objective: To identify practices performed by nurses in the Municipal Health Department of Ribeirão Preto, Brazil, in terms of breastfeeding promotion for pregnant women and/or adolescent mothers. Method: A descriptive and qualitative study was conducted with 12 nurses from July to August 2009 by means of semistructured interviews and observations. The data was analyzed using the content analysis technique. Results: We identified the following categories: "Work based on technique as recommended and biological practices", "Role of the Health service in daily care for pregnant women and/or teenage mothers" and "Professional relationship between nurses and pregnant women and/or adolescent mothers". Nurses face the challenge of changing their procedure-based approach and shifting towards a care provision centered on the patient. They express their concern to develop a trusting relationship with pregnant women and/or teenage mothers based on listening and incorporating social and subjective issues. Conclusion: It is necessary to continuously train and educate in breastfeeding in order to develop a new profile for nurses capable of providing comprehensive care and meeting the needs of adolescents.
\end{abstract}

Key words: Adolescents, comprehensive health care, breastfeeding, nursing.

\title{
RESUMEN
}

Objetivo: Identificar la práctica de las enfermeras que actúan en la red municipal de salud de Ribeirão Preto, Brasil, en relación a la promoción de la lactancia materna para gestantes y/o madres adolescentes. Material y método: Estudio descriptivo, cualitativo, realizado en la red básica de salud de Ribeirão Preto, Brasil, con 12 enfermeras, entre julio y agosto de 2009, por medio de entrevistas semiestructuradas y de observación. Se analizaron los datos de acuerdo con la técnica de análisis de contenido, modalidade temática. Resultados: Identificamos las categorías "trabajo centrado en la técnica, según lo recomendado y el biológico", "el servicio de salud en el cuidado diario para las mujeres embarazadas y/o madres adolescentes" y "relación profesional entre el profesional y la mujer embarazada y/o madre adolescente". Las enfermeras enfrentan en su práctica el desafío de la transformación de la asistencia centrada en el procedimiento para la atención focalizada en el usuario. Enfatizan la preocupación por desarrollar una relación de confianza basada en la escucha y en la incorporación de la dimención social y la subjetiva de las gestantes y/o madres adolescentes. Conclusión: Es necesario la capacitación y educación permanente sobre lactancia materna, procurando un nuevo perfil de los enfermeros para la atención integral de las necesidades de las adolescentes.

Palabras clave: Adolescentes, atención integral de salud, lactancia materna, enfermería.

Fecha recepción: 05/01/15 Fecha aceptación: 25/08/16

\section{INTRODUÇÃO}

A prática do aleitamento materno tem sido defendida e apoiada no mundo todo como a melhor forma de nutrição exclusiva para o bebê até o sexto mês de vida e complementar até o segundo ano de vida. Além de trazer benefícios nutricionais, pelo leite conter todos os nutrientes em qualidade e quantidade necessárias para o desenvolvimento adequado do lactente, o aleitamento materno favorece e intensifica o vínculo entre mãe e filho (1, 2). Apesar de todos os benefícios e vantagens do aleitamento materno serem amplamente conhecidos e divulgados e ainda, do conhecimento técnico-científico acumulado pelos profissionais de saúde, o desmame precoce é uma realidade que prevalece no Brasil (3).

O estudo de Dagher et al. (4) destaca a importância dos profissionais de saúde identificarem grupos-alvo específicos e realiza- 
rem intervenções para promover o início e a manutenção da amamentação. Contudo, não basta que os profissionais tenham a intenção de realizar tais práticas; é preciso que estejam preparados e motivados para atuarem junto aos usuários dos serviços de saúde.

A adolescência é um dos diversos fatores que contribuem para a ocorrência do desmame precoce. Está relacionada ao baixo nível educacional e socioeconômico das adolescentes, à dificuldade de acesso à informações sobre aleitamento materno e à falta de apoio de pessoas significativas a elas e de profissionais de saúde (5).

Os profissionais da saúde, por meio de suas ações e do papel que desempenham, influenciam positiva ou negativamente o início e a duração da amamentação (6). O conhecimento produzido e divulgado até o momento, deixa uma lacuna quanto a como se dá a prática cotidiana dos enfermeiros frente ao que diz respeito ao aleitamento materno. Sabemos que as atividades de promoção, incentivo e apoio são essenciais para o sucesso do aleitamento materno e que o modelo de trabalho atual tende a privilegiar a prática do enfermeiro focada na dimensão biológica em detrimento das demais dimensões humanas (7). O que não sabemos é como se dá a prática do enfermeiro no que tange o aleitamento materno, seu posicionamento frente à gestante e à nutriz e em especial quando esta é uma adolescente.

Entendemos ser premente a importância da realização deste estudo. Neste artigo, nós analisamos a atuação das enfermeiras da atenção básica de saúde de Ribeirão Preto, SP, junto à adolescentes no ciclo gravídico-puerperal especificamente nas questões relativas ao aleitamento materno.

Este estudo objetivou identificar as práticas das enfermeiras que atuam na rede municipal de saúde do município de Ribeirão Preto, SP, sobre a promoção do aleitamento materno de adolescentes no ciclo gravídicopuerperal.

\section{MÉTODO}

Trata-se de um estudo descritivo, com abordagem metodológica qualitativa (8). Considerando os enfermeiros como sujeitos de suas ações em relação ao aleitamento materno, a abordagem qualitativa permite a compreensão da subjetividade expressa nas falas e nos atos acerca das próprias práticas de saúde.

A pesquisa foi realizada no município de Ribeirão Preto, São Paulo, Brasil, tendo como local para identificação e recrutamento das participantes, as Unidades de Saúde do Distrito Sul de Ribeirão Preto, por se constituírem em locais de maior fluxo de atendimentos pré-natal de adolescentes, segundo dados do Departamento de Informática do Sistema Único de Saúde de 2009.

Inicialmente, foi consultado nas Unidades de Saúde participantes do estudo o número de enfermeiras que estavam trabalhando. Concluída esta consulta, o convite para participar da pesquisa foi realizado durante os dias de trabalho das enfermeiras. Dessa forma, participaram do estudo as enfermeiras trabalhadoras das Unidades de Saúde do Distrito Sul que nos informaram ter contato com gestantes e/ou puérperas em sua rotina de trabalho. O tamanho da amostra foi determinado pela saturação dos dados. A saturação ou recorrência dos dados caracteriza-se pelo momento no qual a busca de novos sujeitos não acrescenta mais nenhum dado novo à investigação. Para constatação da saturação teórica utilizamos os seguintes passos: a) disponibilizar os registros de dados "brutos"; b) "imergir" em cada registro; c) compilar as análises individuais (de cada pesquisador, para cada entrevista); d) reunir os temas ou tipos de enunciados para cada pré-categoria ou nova categoria; e) codificar ou nominar os dados; f) alocar, numa tabela, os temas e tipos de enunciados (9). Utilizando-se os critérios descritos, chegamos a um 
universo de 12 enfermeiras. Visando garantir a privacidade e anonimato das informações, as 12 enfermeiras, participantes do estudo estão identificadas por números ordinais.

O primeiro contato ocorreu após a aprovação do projeto de pesquisa pelo Comitê de Ética, protocolo No 0983/2009 e pela Secretaria Municipal da Saúde de Ribeirão Preto, SP, em respeito aos preceitos éticos da Resolução 466 de 12/12/2012, do Conselho Nacional de Saúde, sobre pesquisas envolvendo seres humanos. Neste encontro foram explicados os objetivos da pesquisa, sua finalidade e foi firmado um acordo para a realização da observação participativa. Neste momento, todas as participantes assinaram o termo de consentimento livre e esclarecido.

Os dados foram coletados pela pesquisadora durante os meses de julho e agosto de 2009. Utilizamos a técnica de entrevista semiestruturada (8), realizadas em local reservado nas dependências das Unidades de Saúde e áudio-gravadas, de modo a favorecer o diálogo entre as participantes e o entrevistador. Foram realizados dois encontros entre os mesmos e a média de duração das entrevistas foi de vinte e três minutos. Utilizamos um roteiro de perguntas com dados sociodemográficos, profissional e de trabalho e questões sobre a prática do enfermeiro em relação à promoção do aleitamento materno para gestantes e/ou mães adolescentes. Buscando fazer uma leitura do fenômeno o mais próximo possível da realidade vivenciada pelos sujeitos pesquisados, utilizamos a observação de campo cujos dados acerca do contexto foram registrados em um diário de campo e utilizados na análise, como dados complementares às entrevistas (8).

Na Unidade de Saúde, a observação iniciava no momento em que a adolescente chegava à sala de espera e finalizava quando a mesma saía da sala de consulta. As observações foram realizadas considerando-se todos os acontecimentos, na sala de espera e de consulta e domicílio, relacionados com a prática das enfermeiras que participaram do estudo.
No domicílio, a pesquisadora acompanhava a enfermeira desde sua chegada até a saída.

A análise das informações foi fundamentada na técnica de análise de conteúdo, modalidade temática, a qual consiste em descobrir os núcleos de sentido que compõem uma comunicação, cuja presença ou frequência acrescentem significado ao objeto de estudo. O material foi transcrito e analisado seguindo as três fases do método. Na primeira fase, denominada de pré-análise, se buscou fazer uma leitura exaustiva dos dados, seguida da organização dos dados e da formulação de hipóteses. Na sequência, foi realizada a exploração do material onde buscou-se codificar, classificar e agregar os dados brutos. $\mathrm{Na}$ terceira e última fase, os dados foram interpretados e delimitados em temas, de acordo com os significados atribuídos (8).

\section{RESULTADOS}

O conteúdo das entrevistas nos possibilitou identificar três unidades temáticas: "trabalho centrado na técnica, no recomendado e no biológico", "cotidiano do serviço de saúde na atenção às gestantes e/ou mães adolescentes" e "relação profissional de saúde e gestante e/ ou mãe adolescente".

\section{Trabalho centrado na técnica, no recomendado e no biológico}

O trabalho de promoção e apoio ao aleitamento materno realizado pelas enfermeiras no atendimento a gestantes e/ou mães adolescentes revelou-se focalizado na dimensão biológica. O olhar para as mamas da mulher que amamenta, a preocupação com a orientação da condução correta da mamada, o manejo baseado no protocolo da Secretaria Municipal da Saúde, mostra-nos uma atuação distante da integralidade, ainda que pertinente e fiel ao modelo vigente. 
Quando a mãe fala que está tudo bem [...] algumas mães chegam aqui e realmente está bem: a mama está boa, não esta túrgida, né, não tem fissura não tem rachadura, né [...] Então, a gente dá uma reforçada nas orientações [...]. (P2) Às vezes, vem alguém da família trazer, no teste do pezinho e não é a mãe. Eu falo: não, tem que vir [...] nós precisamos ver como é que tá essa mama. (P7)

Entretanto, uma enfermeira relatou-nos que no seu dia a dia de trabalho, prioriza a proximidade com a adolescente, buscando conhecer a usuária adolescente em suas várias dimensões enquanto ser social.

Percebo muitas vezes, que a adolescente não tem o apoio da família, ou não tem o parceiro fixo, né está mais susceptível [...] Então, neste momento, são aspectos emocionais e sociais mais presentes do que problema com o aleitamento em si. Porque, por conta desses fatores, ela vai estar mais fragilizada, ela corre um risco maior de deixar a amamentação. (P1)

A prática educativa em aleitamento materno utiliza o modelo preventivo de educação em saúde, com apresentação de temas preestabelecidos e apoio de materiais educativos.

Existe o grupo de gestante [...] eu sei que elas passam vídeos, elas conversam, são várias aulas, são todas assim [...] aulas agendadas mesmo. Então, é um grupo já específico, com determinadas aulas fechadas. (P2)

[...] a gente tem materiais informativos, né, que são distribuídos e eu pego esses materiais e falo de um assunto muito especial [...] e vou abrir todo o material informativo, desenhos, vou mostrando as alterações que vão acontecendo [...]. (P3)

Quanto à fundamentação teórica e científica para as práticas educativas, as enfermeiras expressaram a necessidade de pautar-se em protocolos e fluxos dos programas da
Secretaria Municipal da Saúde, baseados em normativas do Ministério da Saúde, Secretaria de Estado da Saúde e artigos científicos, como, por exemplo, para fazer as orientações em relação aos cuidados com as mamas.

Olha, a nossa conduta é [...] a gente segue sempre o protocolo da secretaria, é a ordenha manual e o banho de sol, tá, e aí no dia-a-dia a gente vai avaliando pra saber se tem que fazer a suspensão da amamentação [...] por causa de escoriação do mamilo [...]. (P10)

Foi possível identificar outras informações que são transmitidas às gestantes e/ou mães adolescentes revelando uma dualidade entre práticas recomendadas e não recomendadas pelo Ministério da Saúde e Secretaria Municipal da Saúde.

Durante a primeira consulta, né [...] a gente orienta já pra manter o seio prá dar uma escovação né, na mama, prá manter o mamilo mais flexivel [...] depois no puerpério é que a gente vai orientar, a hora que ela vier fazer o teste do pezinho, a apojadura, o banho de sol, a ordenha manual. (P12)

A gente orienta [...] tomar sol nas mamas, né, explica [...] lavagem das mamas, não usar creme nos mamilos, né, é [...] quando for tomar banho, usar a buchinha prá lavar com uma compressão mais de lado, né [...] pra que a pele vá tomando uma consistência mais resistente, né. (P3)

\section{Cotidiano do serviço de saúde na atenção às gestantes e/ou mães adolescentes}

No dia a dia do trabalho, as enfermeiras contam com parcerias intra e extra institucionais e se mobilizam de forma interdisciplinar na busca de outros saberes para complementarem suas ações.

[...] E em relação ao outro leite, eu sempre encaminho pro pediatra pra dar uma avaliada, né. Por que é que está introduzindo, né [...] então, 
eu faço minhas orientações, mas converso com o pediatra também. (P2)

Porém, na organização do processo de trabalho, a comunicação entre os profissionais ocorre de forma burocrática, por meio de agendamento ou encaminhamento para o profissional de outra categoria, surgindo, assim, o reconhecimento das fragilidades nas relações profissionais, tais como a dificuldade ou inexistência do trabalho em equipe para atendimento à gestante e/ou mãe adolescente.

[...] a gestante chega e [...] a gente vai atuando [...] não existe assim [...] um intercâmbio entre o ginecologista e a gente, sabe [...] não existe isso [...]. (P4)

Aqui, você vê assim cada um tem uma atitude frente a um problema novo. Então, por exemplo, uma paciente estava com sinais de mastite, com muita dor, com febre, com hiperemia, com tudo [...] fui lá no médico, pedi para ele se podia medicar, e o médico falou: você acha que vou passar anti-inflamatório para quem está amamentando? (P5)

A atuação da enfermeira se dá por meio de consultas na unidade de saúde e de visitas domiciliares e parece seguir uma rotina e o protocolo.

[...] A gente sempre procura fazer um atendimento agendado para que a gente possa ter tempo disponivel para a pessoa [...] então, a gente faz naturalmente o acolhimento dessa gestante [...] faço o teste de pregnosticon. Eu vou perguntando data da última menstruação, se tem parceiro fixo, se faz uso de algum método [...] para tentar me aproximar dessa gestante. A gente aborda a questão do aleitamento materno pra ela já começar a se cuidar [...] já marca a primeira consulta com o ginecologista e posteriormente, a gente se coloca a disposição dessa adolescente. (P1)

Se ela (adolescente) falta nas consultas de prénatal, a gente faz a busca dessa paciente, quan- do faz a visita tá abordando, né, a importância do pré-natal, já tá abordando os cuidados, [...] e tá abordando ela toda vez que ela procura a unidade, durante as consultas do pré-natal, a questão da amamentação [...]. (P10)

\section{Relação profissional de saúde e gestante e/ ou mãe adolescente}

As enfermeiras revelaram a preocupação em construir uma relação de confiança, disponibilizando-se para a escuta. Respeitam a decisão da adolescente em amamentar ou não e parecem estar dispostas a ouvirem e a orientar as adolescentes acerca do aleitamento materno.

[...] elas acham que nós somos mais experientes então, eu tento nivelar essa relação nesse contato. Dizer que eu não estou julgando, que eu não quero saber, né [...] porque que ela fez, com quem ela fez, como ela fez, mas é nesse sentido de dizer que eu estou disponível para ajudá-la naquele momento. (P1)

Vamos lá, vamos tentar, vamos pegar, vamos colocar o bico, vamos [...] sabe [...] a gente tenta de todas as maneiras e quando a gente percebe que não é a questão que o bico é ruim, que é ela que não quer, mas se esconde atrás do bico que é ruim, aí a gente acha que tem que respeitar, né $[. .$.$] . (P10)$

[... eu acho que não tem uma prática pra adolescente [...] então a gente acaba trabalhando né, de igual mesmo, a gente conversa muito com as adolescentes no sentido de falar da importância (do aleitamento materno). (P10)

As falas das enfermeiras ressaltaram uma tendência à incorporação, ao cotidiano do trabalho, de questões relacionadas ao social ou à subjetividade das gestantes e/ou mães adolescentes. No entanto, desvencilhar dos conceitos pessoais e de julgamentos parece ser uma questão delicada.

Por que que engravidou tão cedo? Ah, engravidou porque não veio, porque não usou preserva- 
tivo ou porque furou ou porque queria [...] tem umas que querem mesmo. Então, depois [...] você vai querer seguido um nenê? você é muito nova, tem que estudar, trabalhar [...]. (P12)

Agora a adolescente [...] muitas querem ter a mesma vida, de sair, de não ter tanto compromisso assim, né. Então, é aquela coisa, quanto mais jovem mais complicado é. (P5)

As enfermeiras relataram que se sentem despreparadas para lidar com os aspectos da amamentação de gestantes e/ou mães adolescentes:

Eu acho que adolescente é muito difícil [...] eu não fui treinada pra lidar com adolescente amamentando, com todas as dificuldades, acho que o serviço não está preparado, acho que nós não estamos preparadas pra lidar, nem com a prevenção, nem com uma gravidez indesejada [...] muito menos com as complicações, porque cada mãe sente muita dificuldade. (P9)

\section{DISCUSSÃO E CONCLUSÃO}

A partir dos resultados desta pesquisa, gostaríamos de discutir alguns pontos considerados essenciais por nós e que ultrapassam as questões inerentes ao aleitamento materno.

O primeiro é o desafio que as enfermeiras enfrentam em sua prática, considerando o modelo de saúde vigente no município, a organização do trabalho e o espaço que é necessário ter para, de fato, desenvolver ações de promoção da saúde. Quando o acolhimento é tomado como um pronto atendimento as necessidades relacionadas às queixas clínicas são questões pontuais, onde as queixas são identificadas como necessidades de saúde, conforme abordagem que privilegia o componente biológico do atendimento clínico, acentuando-se o padrão queixa-conduta, havendo possibilidade de se perpetuar a exclusão das adolescentes ao sistema de saúde, dificultando a interação entre a adolescente e o profissional $(10,11)$.

No que diz respeito ao serviço de saúde, é fundamental que a adolescente busque o serviço e compreenda que o profissional da saúde é um mediador importante no cuidado à sua saúde. Concordamos com Santos e Ressel (12) pois a maneira com que a adolescente é recebida no serviço de saúde pode cativála ou simplesmente afastá-la imediatamente da busca pelo atendimento. Dessa forma, a acolhida das adolescentes ao serviço de saúde deve ser cordial, compreensiva, de forma que as mesmas se sintam valorizadas e tenham confiança no profissional que as recebe (12).

O desafio que se coloca é a transformação da atenção sanitária centrada no procedimento em uma atenção centrada no usuário, por meio do acolher, do vínculo, das ações de responsabilização entre profissionais e adolescentes e das ações de estímulo à autonomia da adolescente (13).

O modelo de atenção integral tem por objetivo ampliar a possibilidade de atuação do profissional, tendo em vista não só a singularidade do sujeito, mas também a organização do serviço que lhe é prestado. Neste modelo, a saúde é entendida como um requisito para a cidadania e envolve inter-relações entre o indivíduo, a coletividade e o meio ambiente (12).

O segundo ponto é o cotidiano de trabalho das enfermeiras, sob a ótica do processo de trabalho, em suas práticas de promoção ao aleitamento materno para gestantes/mães adolescentes. Nosso estudo evidenciou que a comunicação entre profissionais de diferentes categorias ocorre de forma burocrática e impessoal. Desta maneira, a oferta de serviços à usuária fragiliza-se mediante a forma hierárquica de relação profissional, tais como a dificuldade ou inexistência do trabalho em equipe para atendimento à gestante e/ou mãe adolescente.

Contrapondo-se a este resultado, o estudo de Costa et al. (14) informa que as ações integradas entre os profissionais que atendem as adolescentes certamente facilitam o 
alcance da integralidade da atenção. Nesse sentido, para que a integralidade do cuidado seja realizada na prática profissional é necessário que os profissionais atuem de maneira interdisciplinar, focalizando as necessidades das adolescentes, promovendo a assistência qualificada e voltada para o acolhimento, o vínculo e o acesso (13).

A busca do trabalho inter e multiprofissional deve ser uma constante pelos profissionais; e os obstáculos presentes no cotidiano de suas práticas não devem se constituir em um impedimento à integralidade da atenção. Em um serviço de saúde todas as categorias profissionais podem se qualificar para o atendimento de adolescentes e jovens. A atuação de profissionais de diversas áreas favorece a troca de ideias e proporciona educação e apoio junto às adolescentes, formando um saber capaz de dar conta da complexidade dos problemas e necessidades de saúde das adolescentes $(14,15)$.

O terceiro e último ponto apresentado nesta discussão é a relação das enfermeiras com a usuária, em nosso estudo, a gestante/ mãe adolescente. As enfermeiras ressaltaram sua preocupação em desenvolver uma relação de confiança, baseada na escuta, bem como em incorporar questões relacionadas a outras dimensões como o social e o subjetivo das gestantes e/ou mães adolescentes.

Para isso, a chave é individualizar um enfoque pessoal para necessidades específicas, identificadas a partir do conhecimento tanto do indivíduo como do seu ambiente social.

É necessário que a rede básica de saúde assegure um espaço no qual a usuária seja vista na sua integralidade e de fato apoiada em suas decisões. Significa capacitá-la por meio do diálogo e de reflexões para solucionar os problemas do cotidiano. Isto implica em respeitar as limitações e saberes prévios da adolescente e compartilhar das vivências de sua realidade (16). Isto implica na criação de estratégias que possibilite conhecê-las em seus projetos de vida, de maneira integrada com sua família e meio social, tornando-as protagonistas das vivências em amamentação, por meio da construção de vínculo e do acolhimento visando garantir uma atenção integral (17).

As práticas educativas em aleitamento materno podem ser desenvolvidas em um contexto, o de vida das adolescentes, em que vários profissionais estejam inseridos, e da articulação interdisciplinar que se proporciona melhor qualidade da atenção em saúde (18).

Os profissionais de saúde precisam ser mais capacitados para trabalhar com a promoção do aleitamento materno junto a adolescentes, seja por meio de instituições de ensino e formação, seja por meio de gestores da saúde, a fim de consolidar equipes multiprofissionais integradas e comprometidas com a saúde materna e infantil (19).

As demandas atuais em saúde implicam em desafios para a formação de recursos humanos que ultrapassem os limites da educação formal. Ganha relevância a educação permanente em saúde, que parte do pressuposto da aprendizagem significativa e propõe a transformação das práticas profissionais, baseada na problematização do processo de trabalho em um processo de reflexão crítica sobre as práticas reais, com pessoas que vivenciam juntas uma experiência ou trabalho em saúde em direção à integralidade (11).

Acreditamos que os resultados deste estudo correspondem à realidade atual em nosso país, apesar do intervalo decorrido desde a realização das entrevistas e concluímos que são necessárias capacitação e educação permanentes em aleitamento materno, visando a um novo perfil de enfermeiros para a atenção integral das necessidades das adolescentes, no ciclo gravídico-puerperal.

\section{REFERÊNCIAS}

1. Kramer M, Kakuma R. The optimal duration of exclusive breastfeeding: a syste- 
matic review [Internet]. Geneva: World Health Organization; 2002 [citado 12 mar 2016]. 47 p. Disponível em: http:// www.who.int/nutrition/publications/ optimal_duration_of_exc_bfeeding_review_eng.pdf

2. Passanha A, Benício MHD, Venâncio SI, Reis MCG. Implementation of the Brazilian Breastfeeding Network and prevalence of exclusive breastfeeding. Rev. Saúde Públ. 2013; 47(6): 1141-1148.

3. Vieira TO, Vieira GO, Oliveira NF, Mendes CMC, Giugliani ERJ, Silva LR. Duration of exclusive breastfeeding in a Brazilian population: new determinants in a cohort study. BMC Pregnancy Childbirth [Internet]. 2014 [citado 12 mar 2016]; 14: 175-184. Disponível em: https://bmcpregnancychildbirth.biomedcentral. com/articles/10.1186/1471-2393-14-175

4. Dagher RK, McGovern PM, Schold JD, Randall XJ. Determinants of breastfeeding initiation and cessation among employed mothers: a prospective cohort study. BMC Pregnancy Childbirth [Internet]. 2016 [citado 12 dic 2016]; 16(1): 194-204. Disponível em: https://www. ncbi.nlm.nih.gov/pubmed/27472915

5. Alves VH, Rodrigues DP, Gregório VRP, Branco MBLR, Souza RMP, Alves CMCSH. Reflexões sobre o valor da amamentação como prática de saúde: uma contribuição da enfermagem. Texto Contexto Enferm. 2014; 23(1): 203-10.

6. Apostolakis-Kyrus K, Valentine C, DeFranco E. Factors associated with breastfeeding initiation in adolescent mothers. J Pediatr. 2013; 163(5): 1489-94.

7. Horta BL,Victora CG.Long-term effects of breastfeeding: a systematic review [Internet]. Geneva: World Health Organization; 2013 [citado 12 mar 2016]. 69 p. Disponível em: http://apps.who.int/iris/bitstream/10665/79198/1/9789241505307_eng. pdf

8. Minayo MCS. O desafio do conhecimento: pesquisa qualitativa em saúde. 11 ed.
São Paulo: Hucitec; 2008. 407 p.

9. Fontanella BJB, Luchesi BM, Saidel MGB, Ricas J, Turato ER, Melo DG. Amostragem em pesquisas qualitativas: proposta de procedimentos para constatar saturação teórica. Cad Saude Publica. 2011; 27(2): 388-94.

10. Ayres JRCM, Carvalho YM, Nasser MA, Saltão RM, Mendes VM. Caminhos da integralidade: adolescentes e jovens na Atenção Primária à Saúde. Interface Comunic., Saude, Educ. [Internet]. 2012 [citado 12 mar 2016]; 16(40): 67-81. Disponível em: http://www.scielo.br/pdf/ icse/v16n40/aop2212.pdf

11. Mitre SM, Andrade EIG, Cotta RMM. Avanços e desafíos do acolhimento na operacionalização e qualificação do Sistema Único de Saúde na Atenção Primária: um resgate da produção bibliográfica do Brasil. Cienc Saúde Colet. 2012; 17(8): 2071-85.

12. Santos CC, Ressel LB. O adolescente no serviço de saúde. Adolesc Saúde. 2013; 10(1): 53-5.

13. Costa RF, Queiroz MVO, Zeitoune RCG. Cuidado aos adolescentes na atenção primária: perspectivas de integralidade. Esc Anna Nery. 2012; 16(3): 466-72.

14. Costa RF, Zeitoune RCG, Queiroz MVO, García CIG, García MJR. Redes de apoio ao adolescente no contexto do cuidado à saúde: interface entre saúde, família e educação. Rev. esc enferm USP. 2015; 49(5): 741-7.

15. Masson N, Falcão A, Velo MMAC, Pereira AC. Acolhimento e vínculo: tecnologias relacionais na produção da saúde. Rev Bras Pesq Saúde. 2015; 17(2): 103-10.

16. Freire P. Pedagogia da autonomia: saberes necessários às práticas educativas. $43 \mathrm{ed}$. São Paulo: Paz e Terra; 2011. 144 p.

17. Camarotti CM, Nakano MAS, Pereira CR, Medeiros CP, Monteiro JCS. Perfil da prática da amamentação em grupos de mães adolescentes. Acta Paul Enferm [Internet]. 2011 [citado 12 mar 2016]; 24(1): 
55-60. Disponível em: http://www.scielo. br/pdf/ape/v24n1/v24n1a08.pdf

18. Barbosa LN, Santos NC, Moraes MAM, Rizzardi SD, Corrêa EC. Prevalência de práticas educativas acerca do aleitamento materno exclusivo (AME) em Cuiabá -
MT. Esc Anna Nery. 2015; 19(1): 147-53. 19. Almeida JM, Luz SAB, Ued FV. Apoio ao aleitamento materno pelos profissionais de saúde: revisão integrativa da literatura. Rev Paul Pediatr. 2015; 33(3): 355-62. 